\title{
EFECTO DE LA TEMPERATURA DE SUSTRATO SOBRE LA ESTRUCTURA Y RESISTIVIDAD ELÉCTRICA DE PELÍCULAS DELGADAS DE NITRURO DE MOLIBDENO
}

\author{
José Noé Valdivia Rodas ${ }^{\mathrm{a}^{*}}$, Arturo Fernando Talledo Coronado ${ }^{\mathrm{b}}$, Segundo Jáuregui Rosas ${ }^{\mathrm{a}}$, \\ Pedro De La Cruz Rodrígueza, Manuel Enrique Guevara Vera ${ }^{a}$
}

\begin{abstract}
RESUMEN
Películas delgadas de nitruro de molibdeno fueron depositadas sobre obleas de silicio (111) mediante la técnica de la pulverización catódica magnética reactiva $\mathrm{DC}$, a la temperatura de sustrato de $100,200,300$ y $400{ }^{\circ} \mathrm{C}$, en la mezcla de gases $\left(A r+N_{2}\right)$ a la presión de trabajo de $4,3 x^{-3}$ torr. La composición de las películas ha sido definida con espectroscopia Auger (AES). La difracción de rayos X muestra que tales películas presentan una orientación cristalográfica preferencial a lo largo del plano (112) y el tamaño de grano se incrementa desde 8,21 a 13,16 nm en el rango de 100 a $400{ }^{\circ} \mathrm{C}$ de la temperatura de sustrato. La resistividad de las películas disminuye con el aumento de la temperatura de sustrato desde 74,20 a $2,45 \mu \Omega$.cm mostrando características óhmicas. El valor más bajo de la resistividad eléctrica fue de 2,45 $\mu \Omega$. $\mathrm{cm}$ a la temperatura de sustrato de $400{ }^{\circ} \mathrm{C}$.
\end{abstract}

Palabras clave: películas delgadas, nitruro de molibdeno, Difracción de Rayos X, resistividad eléctrica, temperatura de sustrato.

\section{EFFECT OF SUBSTRATE TEMPERATURE ON THE STRUCTURE AND ELECTRICAL RESISTIVITY OF THIN FILMS OF MOLYBDENUM NITRIDE}

\begin{abstract}
Thin films of molybdenum nitride were deposited on silicon wafers (111) by means of the DC reactive magnetic sputtering technique, at the substrate temperature of 100, 200, 300 and $400{ }^{\circ} \mathrm{C}$, in the gas mixture $\left(A r+N_{2}\right)$ at the working pressure of $4,3 x^{-3}$ torr. The composition of the films has been defined with Auger Electron Spectroscopy (AES). X-ray diffraction shows that such films have a preferential crystallographic orientation along the plane (112) and the grain size increases from 8,21 to $13,16 \mathrm{~nm}$ in the range of 100 to $400{ }^{\circ} \mathrm{C}$ the substrate

\footnotetext{
a Facultad de Ciencias Físicas y Matemáticas, Universidad Nacional de Trujillo, Av. Juan Pablo II s/n, Trujillo, Perú.

b Facultad de Ciencias, Universidad Nacional de Ingeniería, Av. Túpac Amaru s/n Rímac, 1301 Lima, Perú.
} 
temperature. The resistivity of the films decreases with increasing substrate temperature from 74,20 to $2,45 \mu \Omega$.cm showing ohmic characteristics. The lowest value of the electrical resistivity was $2,45 \mu \Omega$.cm at the substrate temperature of $400{ }^{\circ} \mathrm{C}$.

Key words: Thin films, molybdenum nitride, $X$-ray diffraction, electrical resistivity, substrate temperature.

\section{INTRODUCCIÓN}

Los nitruros y carburos de metales de transición son estructuras metálicas compactas en las que átomos de carbón o nitrógeno ocupan sitios intersticiales. La unión en su estructura involucra contribución simultáneas de enlace covalente, iónico y metálico para la energía cohesiva, que conduce a propiedades muy interesantes ${ }^{1}$.

Nitruro de molibdeno es uno de esos materiales que exhibe interesantes propiedades. Las películas delgadas de nitruro de molibdeno muestran alta conductividad eléctrica y estabilidad química y son candidatos prometedores para uso como barreras de difusión de $\mathrm{Cu}$ y electrodos en microelectrónica².

También, la película de Mo es una capa de barrera mejor que la película de TiN en prevención de la difusión del cobre hacia el óxido de silicio ${ }^{3}$. Para garantizar alta eficiencia de un dispositivo de célula solar basado en cobre-indio-(galio)-selenio (CIGS), se necesita un contacto óhmico posterior ideal para un mejor transporte de portadores de carga mayoritarios y baja recombinación de portadores de carga minoritarios ${ }^{4}$. Asimismo, células solares $\mathrm{CdTe}$ con nitruro de molibdeno en la estructura de contacto posterior poseen de manera consistente un mejor rendimiento en comparación a células con únicamente molibdeno en el contacto posterior $^{5}$.

Además, nitruro de molibdeno muestra excelente dureza, capacidad catalítica y efectos de superconductividad. Diferentes métodos han sido probados para fabricación de capas de nitruro de molibdeno, incluyendo deposición de capa atómica, deposición asistida por iones, deposición de láser pulsado, implantación fuerte de iones nitrógeno, deposición de vapor química y nitruración de metal Mo a alta temperatura. En comparación con otros métodos, deposición por pulverización catódica reactiva parece ser una de las más efectivas para la fabricación de películas de óxido de metal de transición o de nitruro porque la composición química y de fase de la película depende mucho del Ar-O- $\mathrm{N}_{2}$. En cuanto al método de pulverización catódica magnetrón reactiva, se pueden crear películas de alta calidad incluso a relativamente baja temperatura de sustrato debido a la estimulación del plasma de la interacción iónica con la superficie del sustrato ${ }^{6}$.

Thakur et al. ${ }^{4}$ reportan que la resistividad de películas delgadas de molibdeno sobre vidrio de cal sodada, zafiro, cuarzo y zirconia estabilizada con ytria (YSZ) usando pulverización catódica magnetrón dc a $400{ }^{\circ} \mathrm{C}$ muestran un comportamiento puramente metálico. 
Asimismo, Chen et $a .^{7}$ han encontrado que incrementando la potencia de pulverización catódica DC de películas delgadas de molibdeno desde 200 a $500 \mathrm{~W}$ a 10 mTorr resulta una disminución en la resistividad desde $78,09 \mu \Omega$. $\mathrm{cm}$ a $31,35 \mu \Omega$. $\mathrm{cm}$ debido posiblemente a los cambios de tensiones residuales esfuerzos de tensión a compresión.

Las películas delgadas de molibdeno y nitruro de molibdeno se han utilizado ampliamente en varias áreas tecnológicas, y se han convertido en el paradigma de la tecnología actual y son uno de los temas más importantes para los grupos de investigación de punta. Nuestro objetivo en este trabajo fue analizar el efecto de la temperatura de sustrato sobre la estructura y resistividad eléctrica de películas delgadas de nitruro de molibdeno. La mezcla de gas reactivo fue optimizada para obtener la fase $\mathrm{Mo}_{2} \mathrm{~N}$ como componente principal de la película. La resistividad eléctrica de las películas delgadas de $\mathrm{Mo}_{2} \mathrm{~N}$ fue medido con el método de la probeta de cuatro puntas ${ }^{8}$.

\section{DETALLES EXPERIMENTALES}

\section{Objeto de estudio}

Las películas delgadas de nitruro de molibdeno obtenidas mediante pulverización catódica magnética reactiva DC sobre sustratos de silicio (111) y su variación de la estructura y resistividad eléctrica como respuesta a la temperatura de sustrato.

La población de estudio en la presente investigación fue de 5 películas delgadas, 4 muestras fueron películas delgadas de nitruro de molibdeno y una película delgada de molibdeno puro.

\section{Materiales}

El material que se utilizó fue: Blanco de molibdeno con 7,62 cm de diámetro; 0,64 cm de espesor y 99,97 \% de pureza para pulverización catódica; sustratos de silicio (111), gas argón y nitrógeno con una pureza de 99,99 \%. La generación de las películas delgadas de nitruro de molibdeno se realizó mediante un sistema de pulverización magnética reactiva DC. Para las mediciones eléctricas se utilizó una sonda de cuatro puntas en conjunto con un nanovoltímetro Modelo Keithley 2182 A y una fuente de corriente modelo Keithley 6221 A.

\section{Síntesis de las películas delgadas}

Antes de la deposición, los sustratos de silicio (111), de 2,9 cm x 1,5 cm cada uno fueron limpiados ultrasónicamente en una solución de alcohol de $96^{\circ}$ durante 8 minutos y secado con aire caliente.

En el primer ensayo, se dispusieron varios sustratos de silicio (111) en cado uno de los tres compartimientos de un portasustrato, cada compartimiento estaba separado y debidamente protegido, en dos compartimientos para ser depositado nitruro de molibdeno a $400{ }^{\circ} \mathrm{C}$ y en el otro para ser depositado molibdeno puro también a $400^{\circ} \mathrm{C}$, y luego fueron introducidos en la cámara de vacío de un sistema de pulverización catódica magnética reactiva $\mathrm{DC}$, figura 1 . La distancia del sustrato al blanco de molibdeno fue de $8 \mathrm{~cm}$. La cámara de vacío fue inicialmente 


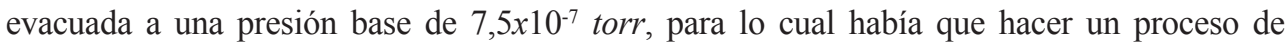
desorción por dos horas, con la finalidad de eliminar la posible formación de vapor de agua en las paredes de la cámara, y la temperatura de sustrato se reguló a $400{ }^{\circ} \mathrm{C}$. Seguidamente, se realizó una pre pulverización catódica durante 2 minutos. Para ello se introduce en la cámara gas

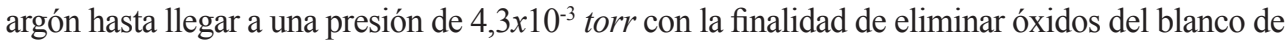
Mo. Luego un flujo de argón fue introducido para mantener la presión durante la deposición a un valor de $4,3 \times 10^{-3}$ torr, y también fue introducido en la cámara de vacío gas nitrógeno a la presión de $2,6 \times 10^{-4}$ torr. La fuente de voltaje se reguló a un valor de $110 \mathrm{~V}$ y con una corriente de 400 $\mathrm{mA}$. La pulverización catódica se realizó durante 10 minutos a uno de los compartimientos que contenía varios sustratos de silicio $(111)$ a $400^{\circ} \mathrm{C}$. Luego externamente se rotaba el portasustrato para una adecuada ubicación del segundo compartimiento que también contenía obleas de silicio (111), al cual se realizó pulverización catódica durante 10 minutos a la temperatura de $400{ }^{\circ} \mathrm{C}$. Seguidamente, se suspendió el flujo de gas nitrógeno y moviendo externamente el portasustrato para una orientación adecuada del tercer compartimiento con sustratos de silicio (111), se depositó molibdeno puro durante 10 minutos a la temperatura de $400{ }^{\circ} \mathrm{C}$.

En ambas muestras la presión de gas argón fue de 4,3 $\times 10^{-3}$ torr y la de nitrógeno de 2,6x10-4 torr. Por lo que se obtuvieron películas delgadas de nitruro de molibdeno y de molibdeno a la temperatura de $400{ }^{\circ} \mathrm{C}$.

En una segunda experiencia, en el mismo portasustrato mencionado anteriormente se dispusieron sustratos de silicio (111) en cada uno de los tres compartimientos, y luego de haber alcanzado

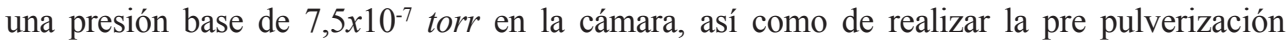
catódica durante 2 minutos; un flujo de gas argón a la presión de 4,3 $\times 10^{-3}$ torr y también gas nitrógeno a la presión de $2,6 \times 10^{-4}$ torr fueron introducidos en la cámara de vacío. Con el mismo valor de voltaje y corriente considerado en el primer ensayo, y regulando la temperatura a $300{ }^{\circ} \mathrm{C}$, se realizó pulverización catódica durante 10 minutos al primer compartimiento del portasustrato. Luego, girando el portasustrato desde la parte externa de la cámara de vacío para lograr una orientación adecuada del segundo compartimiento que contenía varias obleas de silicio (111), y una vez estabilizada la temperatura a $200^{\circ} \mathrm{C}$ y con un flujo de gas argón a la presión de $4,3 \times 10^{-3}$ torr y de gas nitrógeno a la presión de $2,6 \times 10^{-4}$ torr se procedió a la pulverización catódica durante 10 minutos, respectivamente. Finalmente, a una orientación adecuada del tercer compartimiento y después de haber estabilizado la temperatura a $100{ }^{\circ} \mathrm{C}$,

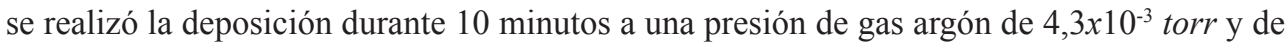

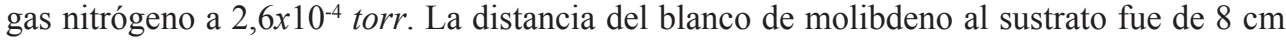
para cada temperatura considerada. Por lo que se obtuvieron películas delgadas de nitruro de molibdeno a las temperaturas de sustrato de 100,200 y $300{ }^{\circ} \mathrm{C}$, respectivamente.

En resumen se generaron películas delgadas de nitruro de molibdeno a 100, 200, 300 y $400{ }^{\circ} \mathrm{C}$ mediante pulverización catódica magnética reactiva $\mathrm{DC}$, usando un blanco de $\mathrm{Mo}$ (7,62 cm de diámetro; 0,64 cm de espesor y pureza de 99,97 \%); así como películas delgadas de molibdeno a la temperatura de $400{ }^{\circ} \mathrm{C}$, a la presión de trabajo de $4,3 \times 10^{-3}$ torr. 


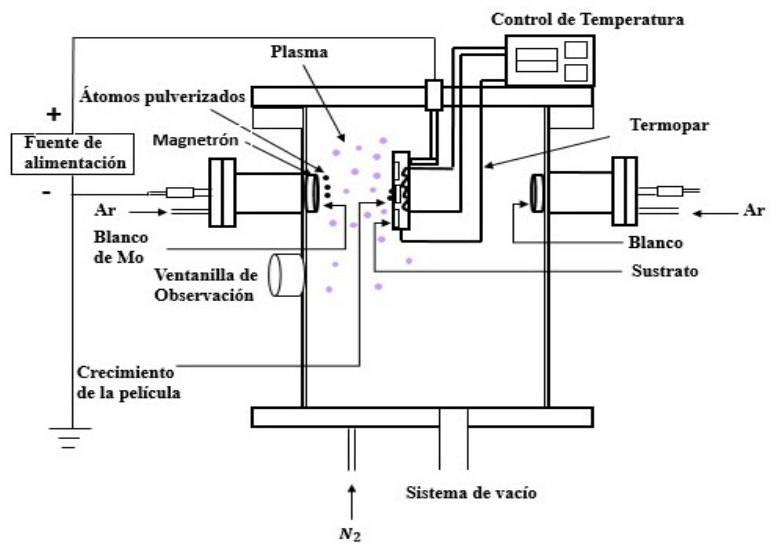

Figura 1. Sistema típico de deposición por pulverización catódica magnética reactiva DC.

\section{Métodos y técnicas}

La caracterización de las muestras fue realizada mediante:

Difracción de Rayos X (DRX). La nanoestructura y orientación cristalográfica de las muestras fueron obtenidas mediante un difractómetro de rayos X Rigaku/Miniflex 600, operado en modo detector scan a $40 \mathrm{kV}, 15 \mathrm{~mA}$, con una fuente de radiación de la lámpara $C u-K \alpha 1$, de longitud de onda $\lambda=1,540 \AA$; , con un tamaño de paso de $0,02^{\circ}$ y un tiempo de conteo de 1s por paso. El tamaño del cristalito (D) fue calculado usando la ecuación de Scherrer ${ }^{9}$ :

$$
D=\frac{0,9 \lambda}{\beta \cos \theta}
$$

donde $\lambda$ es la longitud de onda de los rayos X usado, $\boldsymbol{\beta}$ es el ancho a la altura media del pico de difracción de la muestra y $\theta$ es el ángulo de Bragg.

Técnica de la sonda de cuatro puntas. Para medir la resistividad eléctrica de las películas delgadas se usó la configuración estándar de cuatro puntas, que se muestra en la figura 2.

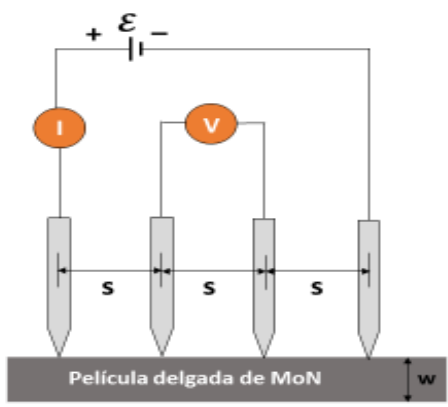

Figura 2. Esquema de la probeta de cuatro puntas para la medida de la resistividad eléctrica; $\varepsilon$ es la fuente de intensidad de corriente, I la corriente aplicada, V la diferencia de potencial eléctrico registrada, w indica el espesor de la película delgada y s la separación entre electrodos ${ }^{10}$. 
Para el caso de una geometría de cuatro puntas lineales con igual espaciado, la resistividad es dada por ${ }^{8}$ :

$$
\rho=\frac{\pi}{\operatorname{Ln} 2} w \frac{V}{I}
$$

donde $\mathrm{w}$ es el espesor de la muestra, I la corriente aplicada y $\mathrm{V}$ el voltaje medido con el nanovoltímetro Modelo Keithley $2182 \mathrm{~A}$.

Espectroscopia electrónica Auger (AES). AES representa hoy la herramienta de análisis químico de superficies más importante para muestras conductoras. La espectroscopia electrónica Auger está basada en el uso de electrones primarios con energías típicas entre 3 y $30 \mathrm{keV}$ y la posibilidad para enfocar y explorar el haz electrónico primario en el rango de micrómetros y nanómetros analizando las capas atómicas más externas de la materia ${ }^{11}$. En el presente trabajo se utilizó dicha técnica para obtener la composición química de las muestras.

La composición de una muestra de n elementos puede ser calculado semicuantitativamente mediante la ecuación ${ }^{11}$ :

$$
x_{A}=\frac{I_{A} / s_{A}}{\sum\left({ }^{I} / s_{i}\right)}
$$

donde $I_{i}$ son las intensidades de los picos elementales y $S_{i}$ son los factores de sensibilidad respectiva, $I_{A}$ la intensidad del pico Auger de un elemento A. Las medidas Auger reportadas en este artículo fueron hechas en el instrumento Riber MIQ 156.

Microscopía de fuerza atómica (AFM). La morfología superficial de las muestras fue realizado mediante un microscopio de fuerza atómica modelo Dimensión Edge AFM-Bruker, con un cantiléver modelo RTESP de material antimonio (n) dopado con Si, de constante elástica $\mathrm{k}=40 \mathrm{~N} / \mathrm{m}$ y frecuencia de $300 \mathrm{kHz}$; con un radio de curvatura de la punta de $8 \mathrm{~nm}$, con una frecuencia de barrido de $10000 \mathrm{~Hz}$.

Microscopía electrónica de barrido (SEM). En un microscopio electrónico de barrido, una pequeña sonda de electrones de 1 a $10 \mathrm{~nm}$ de diámetro escanea en una trama a través de la superficie de la muestra. Los electrones incidentes son dispersados elástica e inelásticamente por el espécimen. La dispersión elástica da como resultado grandes ángulos de dispersión y trayectorias electrónicas en zigzag. Por lo tanto, una fracción de electrones puede dejar la muestra como electrones retro dispersados. La imagen está formada por la señal de los electrones secundarios emitidos, los electrones retro dispersados, los electrones Auger, los cuántos de rayos $\mathrm{X}$, que modulan la intensidad de un tubo de rayos catódicos rastreados en forma sincronizada ${ }^{12}$. Se utilizó dicha técnica para medir el espesor de las películas delgadas de nitruro de molibdeno. El instrumento usado fue HITACHI 8230. 


\section{RESULTADOS Y DISCUSIÓN}

\section{Estructura y composición}

La figura 3 muestra el patrón de DRX de la película delgada de Mo depositada sobre sustrato de $\mathrm{Si}$ (111) preparada a la temperatura de sustrato de $400{ }^{\circ} \mathrm{C}$ mediante pulverización magnética reactiva DC. Un fuerte pico característico de DRX de Mo a $2 \theta=40,58^{\circ}$ es observado correspondiente a la dirección cristalográfica (110), la intensidad del pico de DRX indica que la película crece con una orientación preferencial a lo largo del plano (110); asimismo, el espectro también exhibe otro pico de Mo metálico Mo (211) a $2 \theta \approx 73,7^{\circ}$ de menor intensidad. Chen et al. ${ }^{7}$ reportan picos de difracción similares de películas delgadas de Mo preparadas mediante pulverización catódica dc a diferentes presiones de trabajo. El tercer pico se observa a $2 \theta \approx 28^{\circ}$ el cual corresponde al sustrato de silicio (111). Los datos de DRX coincidieron bien con los archivos JCPDS. El tamaño de cristalito resultó ser de $10,06 \mathrm{~nm}$ calculado del ancho a la altura media del máximo pico (110) usando la ecuación de Scherrer; Kashyout et al. ${ }^{13}$ han obtenido un valor de alrededor de $11 \mathrm{~nm}$ para el tamaño de grano de películas delgadas de Mo crecidas mediante pulverización catódica magnetrón dc a la temperatura de $450{ }^{\circ} \mathrm{C}$. El parámetro de red ha sido calculado en $0,3166 \mathrm{~nm}$.

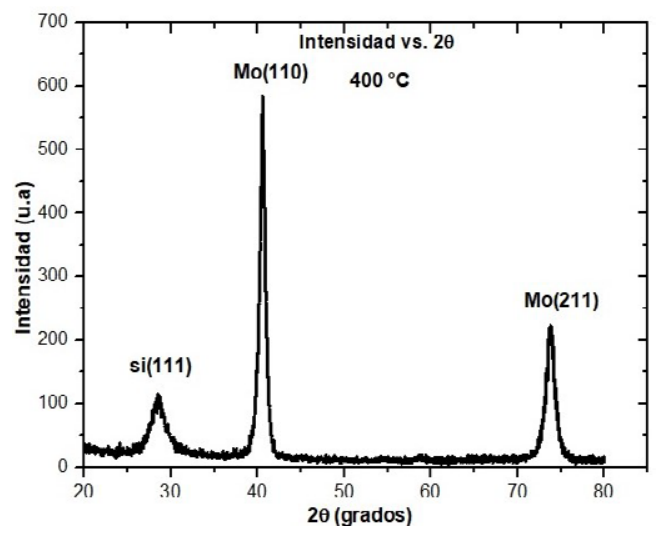

Figura 3. Patrón de DRX de la película delgada de Mo sobre silicio (111) a $400{ }^{\circ} \mathrm{C}$.

La figura 4(a-d) muestra los difractogramas de las películas delgadas de nitruro de molibdeno obtenidas en el rango de 100 a $400{ }^{\circ} \mathrm{C}$, la intensidad de los picos decrecen, y un pico aparece a $2 \theta=37,72^{\circ}$ que es observado en todos los difractogramas, atribuidos a la orientación cristalográfica $\mathrm{Mo}_{2} \mathrm{~N}(112)$, con referencia al International Centre for Diffraction Data 2018, sistema: tetragonal, y grupo espacial: 141/amd (141); Shen et al. ${ }^{14}$ reportan similares picos de difracción para películas delgadas de nitruro de molibdeno preparadas por descarga de arco de a diferentes presiones de nitrógeno. Picos de menor intensidad ocurren a $2 \theta \approx 43,20^{\circ}$ atribuido a la orientación cristalográfica $M o_{2} N(200)$, también a $2 \theta \approx 64,19^{\circ}$ con la orientación cristalográfica $\mathrm{Mo}_{2} \mathrm{~N}(204)$ y el otro pico también de menor intensidad ocurre a $20 \approx 75,59^{\circ}$ atribuido al $\mathrm{Mo}_{2} N(312)$; sistema: tetragonal, y grupo espacial: 141/amd (141), con referencia 
al International Centre for Diffraction Data 2018. Picos de difracción ocurren a $2 \theta \approx 28^{\circ}$ en el rango de temperatura indicado correspondiente al sustrato de $\mathrm{Si}$ (111). A la temperatura de $200{ }^{\circ} \mathrm{C}$ y $400{ }^{\circ} \mathrm{C}$ un pico a aproximadamente $59^{\circ}$ aparece en el espectro, indicando la presencia de partículas de Mo puro ${ }^{15}$, debido probablemente a un aumento en la energía cinética de las partículas de Ar en el plasma. El crecimiento de los cristalitos de las películas delgadas en el intervalo de 100 a $400{ }^{\circ} \mathrm{C}$ de $\mathrm{Mo}_{2} N$ (112), es preferentemente a lo largo del plano (112). El tamaño medio de grano se ha estimado a partir del ancho completo a la mitad del máximo del pico de difracción13 y utilizando la ecuación (1). El tamaño de grano indica un ligero incremento entre 8,21 y $13,16 \mathrm{~nm}$. calculado del ancho completo a la mitad del máximo del pico (112) en el rango de 100 a $400{ }^{\circ} \mathrm{C}$. La película delgada de nitruro de molibdeno $\mathrm{Mo}_{2} \mathrm{~N}$ presenta una estructura tetragonal con parámetro de red: $\mathrm{a}=\mathrm{b}=0,4188 \mathrm{~nm}$; $\mathrm{c}=0,8048 \mathrm{~nm}$.
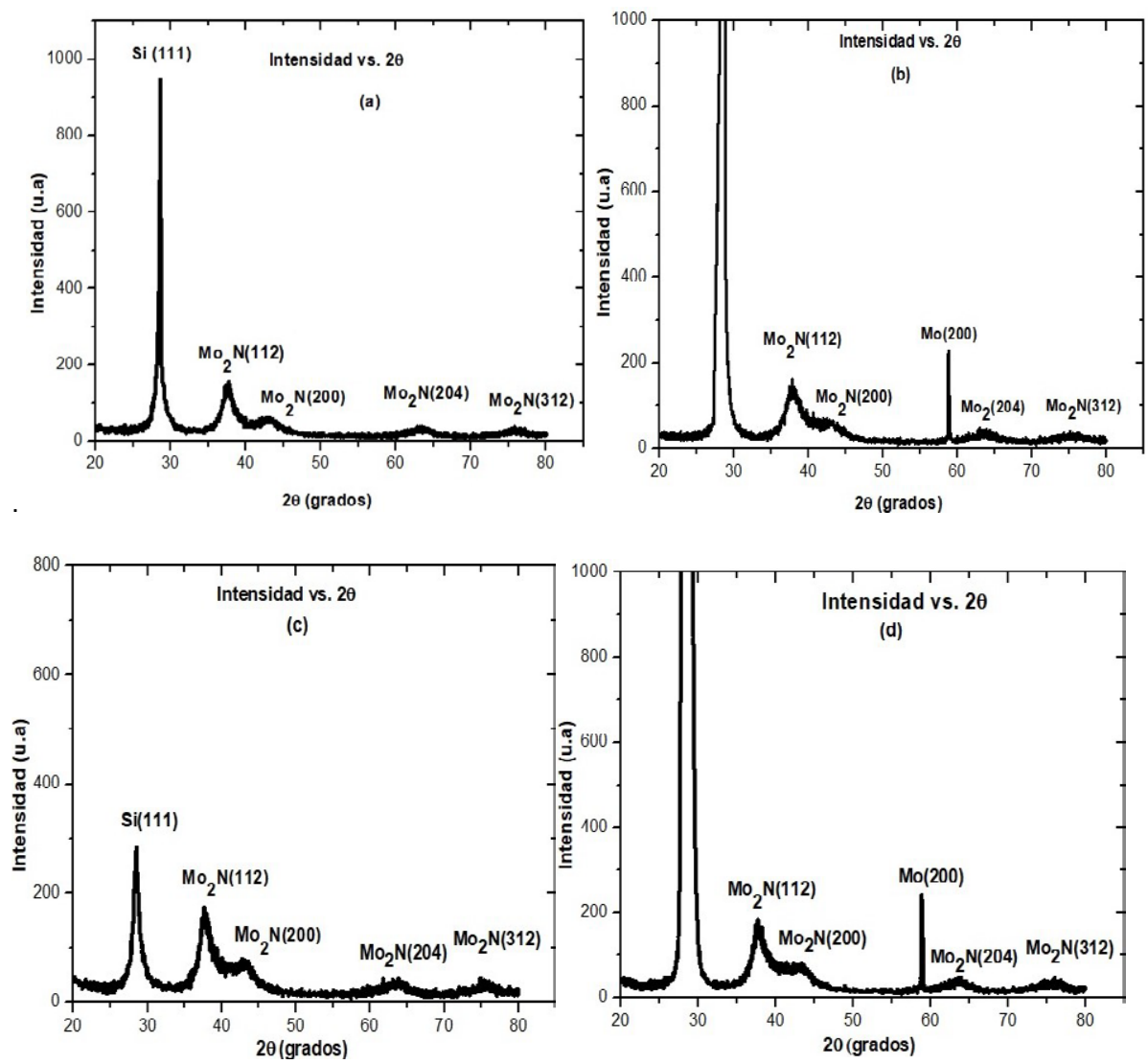

Figura 3. DRX de películas delgadas de $\mathrm{Mo}_{2} \mathrm{~N}$ sobre silicio (111) a diferentes temperaturas de sustrato, (a) $100{ }^{\circ} \mathrm{C}$, (b) $200{ }^{\circ} \mathrm{C}$, (c) $300{ }^{\circ} \mathrm{C}$ y (d) $400{ }^{\circ} \mathrm{C}$. 
En la tabla 1 se resume el análisis estructural de la película delgada de molibdeno y nitruro de molibdeno en función de la temperatura de sustrato.

Tabla 1. Resumen del análisis estructural de la película delgada de $\mathrm{Mo}$ y $\mathrm{Mo}_{2} \mathrm{~N}$ como función de la temperatura de sustrato.

\begin{tabular}{|c|c|c|c|c|}
\hline \multirow{2}{*}{$\begin{array}{l}\text { Muestra } \\
\text { grano }\end{array}$} & 2-Theta & Refl. (hkl) & FWHM / rad & Tamaño de \\
\hline & \multicolumn{3}{|l|}{$( \pm 0,02) /$ grados } & $( \pm 1,18) / \mathrm{nm}$ \\
\hline \multirow[t]{2}{*}{ Mo } & 40,58 & (110) & 0,015 & 10,06 \\
\hline & 73,70 & (211) & & \\
\hline \multirow[t]{4}{*}{$\mathrm{Mo}_{2} \mathrm{~N} 100{ }^{\circ} \mathrm{C}$} & 37,72 & (112) & 0,018 & 8,21 \\
\hline & 43,20 & (200) & & \\
\hline & 64,19 & (204) & & \\
\hline & 75,59 & (312) & & \\
\hline \multirow[t]{4}{*}{$\mathrm{Mo}_{2} \mathrm{~N} 200{ }^{\circ} \mathrm{C}$} & 37,72 & (112) & 0,017 & 8,80 \\
\hline & 43,20 & (200) & & \\
\hline & 64,19 & (204) & & \\
\hline & 75,59 & (312) & & \\
\hline \multirow[t]{4}{*}{$\mathrm{Mo}_{2} \mathrm{~N} 300^{\circ} \mathrm{C}$} & 37,72 & (112) & 0,946 & 10,08 \\
\hline & 43,20 & (200) & & \\
\hline & 64,19 & (204) & & \\
\hline & 75,59 & (312) & & \\
\hline \multirow[t]{4}{*}{$\mathrm{Mo}_{2} \mathrm{~N} \quad 400^{\circ} \mathrm{C}$} & 37,72 & (112) & 0,010 & 13,16 \\
\hline & 43,20 & (200) & & \\
\hline & 64,19 & (204) & & \\
\hline & 75,59 & (312) & & \\
\hline
\end{tabular}

Imagen SEM de sección transversal de una película delgada de $\mathrm{Mo}_{2} \mathrm{~N}$ depositada a $100{ }^{\circ} \mathrm{C}$ es ilustrada en la figura 5. Una densa microestructura con granos columnares de pequeño tamaño desde el sustrato hasta la parte superior de la película es observada, el espesor fue de $260 \mathrm{~nm}$.

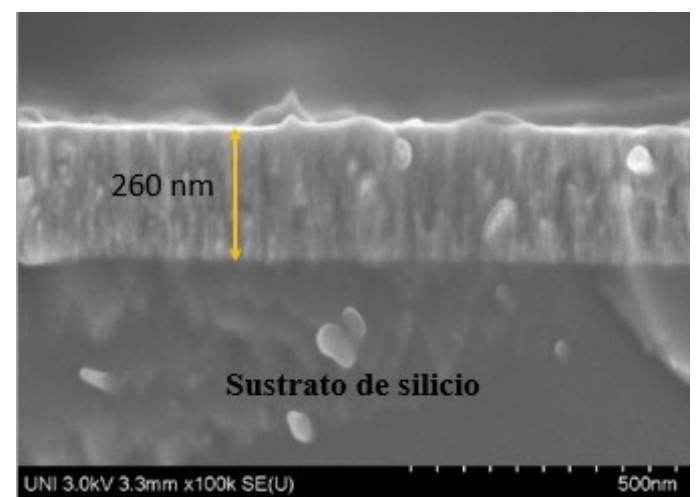

Figura 5. Micrografía SEM mostrando la sección transversal de una película delgada de nitruro de molibdeno depositado a $100{ }^{\circ} \mathrm{C}$ sobre un substrato de silicio. 
El análisis de espectroscopia electrónica Auger de la muestra de $\mathrm{Mo}_{2} \mathrm{~N}$ a $400{ }^{\circ} \mathrm{C}$ indica la presencia de $\mathrm{Mo}_{1}$ y $\mathrm{Mo}$, en los picos con energía de $186 \mathrm{eV}$ y $220 \mathrm{eV}$; así también se observa la presencia de oxígeno, nitrógeno y carbono como se muestra en la figura 6. En la tabla 2 se muestra los resultados de los cálculos.

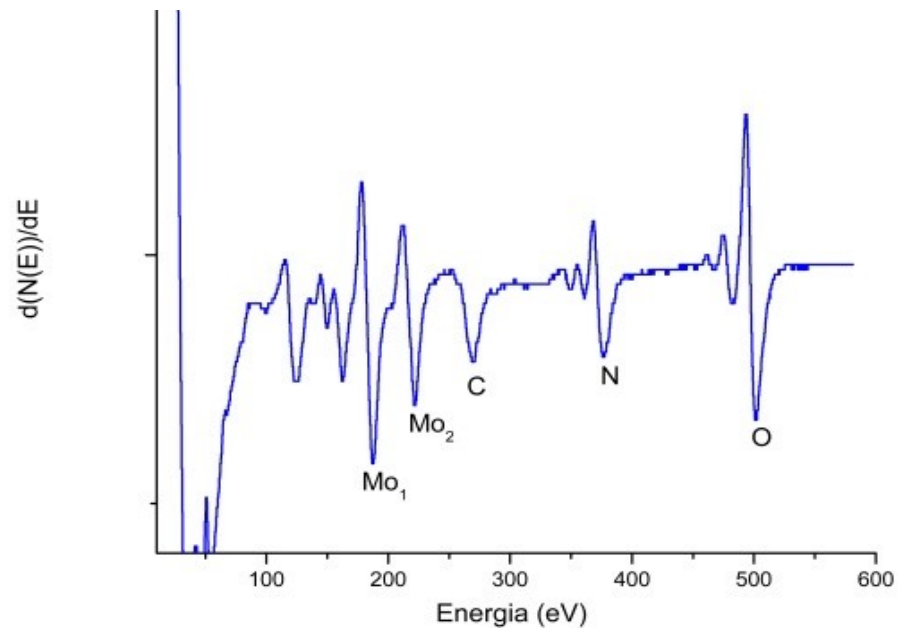

Figura 6. Espectro AES diferencial de una muestra de $\mathrm{Mo} 2 \mathrm{~N}$ a $400^{\circ} \mathrm{C}$ versus energía cinética, donde $\frac{\boldsymbol{d} N(\boldsymbol{E})}{\boldsymbol{d} \boldsymbol{E}}$ revela la oscilación de plasmones.

Tabla 2. Picos Auger y concentraciones de una muestra de nitruro de molibdeno a $400{ }^{\circ} \mathrm{C}$.

\begin{tabular}{ccc}
\hline Elemento químico & Pico Auger /eV & Concentración / (\%) \\
\hline Mo & 186 & 65 \\
$\mathrm{~N}$ & 377 & 35 \\
\hline
\end{tabular}

\section{Propiedades eléctricas}

Los valores de la resistividad eléctrica de las películas delgadas de $\mathrm{Mo}_{2} \mathrm{~N}$ se determinaron con la técnica de la probeta de cuatro puntas 16, los cuales son listadas en la tabla 3. El menor valor de la resistividad fue de 2,45 $\mu \Omega$.cm (la corriente aplicada fue entre 5 a $100 \mathrm{~mA}$ ) a la temperatura de deposición de $400{ }^{\circ} \mathrm{C}$.

Un flujo de corriente I es aplicada entre las probetas externas, y un voltaje $\mathrm{V}$ es medido entre las dos probetas internas ${ }^{17}$. La corriente eléctrica fue suministrada de una fuente de corriente Modelo Keithley 6221, se aplicaron corrientes a cada una de las muestras en el intervalo de 5 a $100 \mathrm{~mA}$, y la lectura de los correspondientes potenciales eléctricos se realizó con un nanovoltímetro Modelo Keithley 2182 A. Con los datos de corriente y voltaje registrados se obtuvo el valor de la resistencia eléctrica (V/I) para las muestras de Mo2N preparadas a 100, 200,300 y $400{ }^{\circ} \mathrm{C}$, respectivamente. 
La medida del espesor de las muestras se realizó mediante microscopía electrónica de barrido, cuyos valores se presentan en la tabla 3. Entonces, utilizando la ecuación (2) se calculó la resistividad eléctrica de las muestras para cada temperatura de sustrato. La resistividad se midió a temperatura ambiente de las muestras obtenidas a diferentes temperaturas de sustrato y los resultados de los cálculos son listados en la tabla 3.

Tabla 3. Valores de la resistividad eléctrica de películas delgadas de nitruro de molibdeno a diferentes temperaturas de sustrato.

\begin{tabular}{cccc}
\hline Temperatura $/{ }^{\circ} \mathrm{C}$ & Resistencia $/ \Omega$ & Espesor $( \pm 2) / \mathrm{nm}$ & Resistividad $/ \mu \Omega . \mathrm{cm}$ \\
\hline 100 & $0,63 \pm 0,08$ & 260 & $74,20 \pm 9,93$ \\
200 & $0,34 \pm 0,03$ & 288 & $43,94 \pm 4,23$ \\
300 & $0,26 \pm 0,02$ & 250 & $29,45 \pm 2,27$ \\
400 & $0,033 \pm 0,001$ & 165 & $2,45 \pm 0,01$ \\
\hline
\end{tabular}

La resistividad de todas la películas delgadas de $\mathrm{Mo}_{2} \mathrm{~N}$ se encuentran en el rango de 74 a $2 \mu \Omega . \mathrm{cm}$, los cuales son mucho menores a los valores reportados de 900 a $500 \mu \Omega . \mathrm{cm}$ de películas delgadas de nitruro de molibdeno crecidas por pulverización catódica magnética reactiva . $^{18}$. La figura 7 muestra la variación de la resistividad eléctrica de las películas con la temperatura de sustrato.

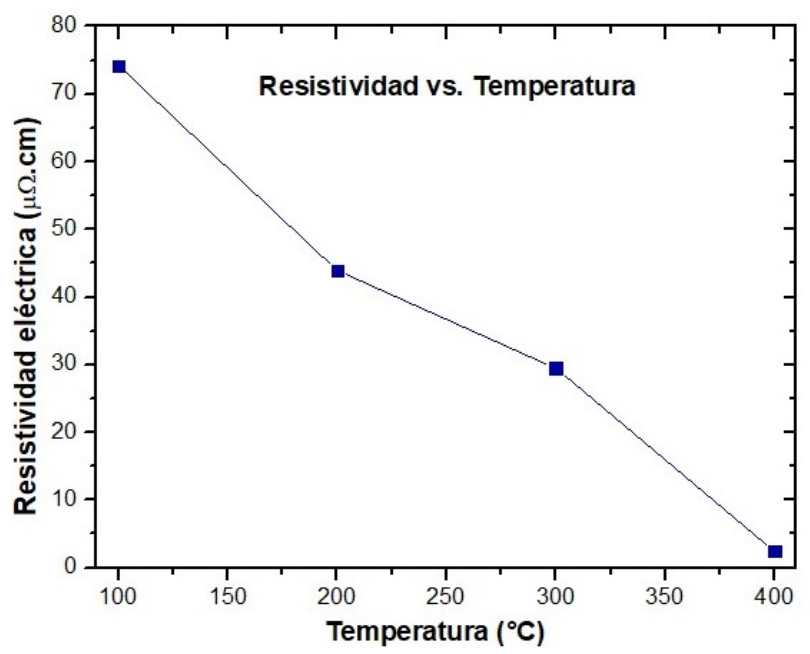

Figura 7. Resistividad eléctrica dependiente de la temperatura de sustrato, medida a temperatura ambiente.

La muestra preparada a la temperatura de $400{ }^{\circ} \mathrm{C}$ tiene un tamaño de grano mayor que la preparada a $100^{\circ} \mathrm{C}$. Este incremento en el tamaño de grano puede ser considerado ser la razón de la disminución en la resistividad de las películas delgadas de $\mathrm{Mo}_{2} \mathrm{~N}$ con el incremento de la temperatura de sustrato ${ }^{19}$. El aumento de temperatura produce incremento de la concentración de portadores, lo cual incrementa la movilidad de los portadores de carga de las películas de 
$\mathrm{Mo}_{2} \mathrm{~N}$ induciendo una gran disminución de la altura de la barrera de potencial de los límites de grano, así como el número de límites de grano. Con el incremento de la temperatura los límites de grano crecen para disminuir la energía de límite de grano, se disminuye las fuerzas cohesivas dentro y a través de los límites. Esto explica los resultados que la muestra preparada con temperatura de sustrato a $400{ }^{\circ} \mathrm{C}$ tiene el menor valor de resistividad eléctrica.

\section{Rugosidad}

La figura 8 presenta micrografías AFM bidimensional (2D) de superficies de $\mathrm{Mo}_{2} \mathrm{~N}$ para varias temperaturas de sustrato, con un área barrida de $5 \mu m \times 5 \mu m$. Se observa una morfología granular en las imágenes AFM. La muestra depositada a $100^{\circ} \mathrm{C}$ exhibe morfología totalmente diferente: una forma ondulada puede ser observada, y la superficie se ha vuelto bastante rugosa debido a la formación de una estructura irregular. Para $200{ }^{\circ} \mathrm{C}$ se observa una disminución de la rugosidad de la superficie de la película. Luego a $300{ }^{\circ} \mathrm{C}$, la superficie es cubierta con suaves capas de nitruro de molibdeno. Sin embargo cuando la temperatura se incrementa a $400{ }^{\circ} \mathrm{C}$, la rugosidad de la superficie se vuelve ligeramente áspera.
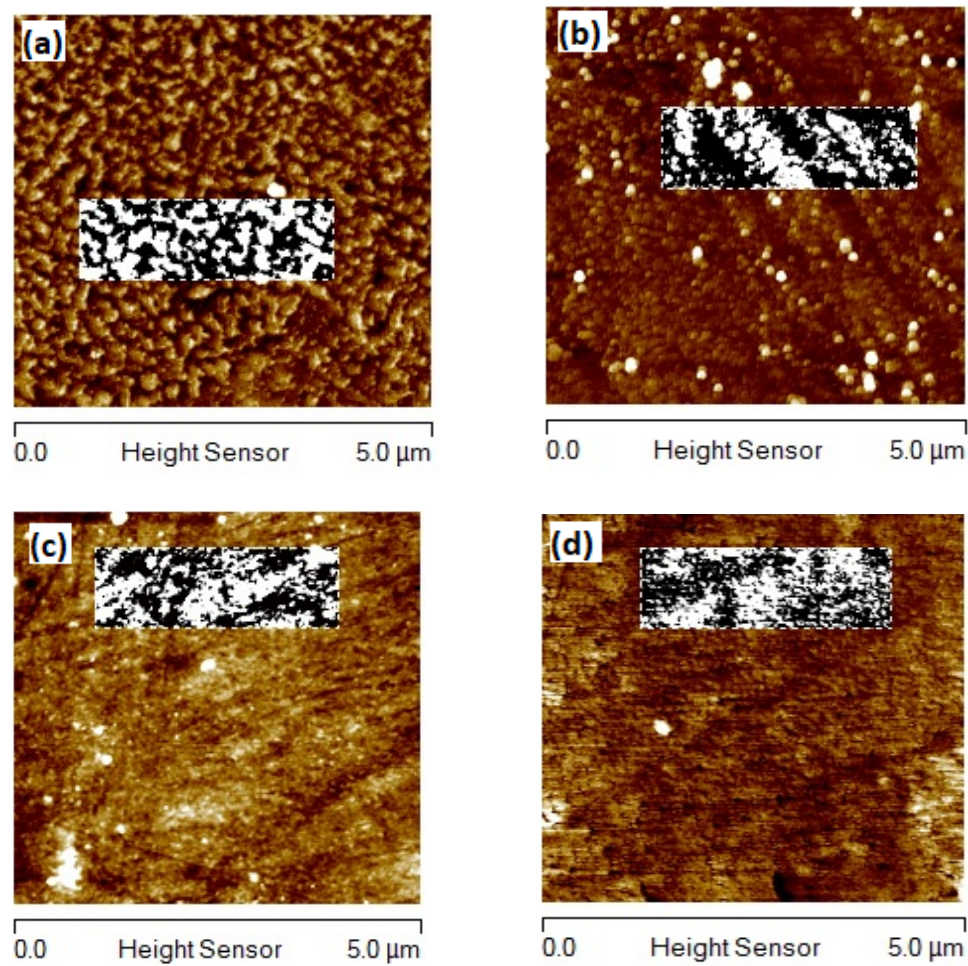

Figura 8. Imágenes AFM 2D de películas delgadas de $\mathrm{Mo}_{2} \mathrm{~N} / \mathrm{Si}$. (a) $100{ }^{\circ} \mathrm{C}$, (b) $200{ }^{\circ} \mathrm{C}$, (c) $300{ }^{\circ} \mathrm{C}$, (d) $400{ }^{\circ} \mathrm{C}$. 
En la figura 9 se presenta una imagen topográfica AFM 3D tomada de un área de $5 \times 5 \mu m$ de la película de $\mathrm{Mo}_{2} \mathrm{~N}$ a $400{ }^{\circ} \mathrm{C}$, mostrando una estructura superficial característica que consiste de partículas de tamaño nanométrico y con una rugosidad de 0,776 $\mathrm{nm}$. La muestra fue preparada a la presión de $4,3 \times 10^{-3}$ torr.

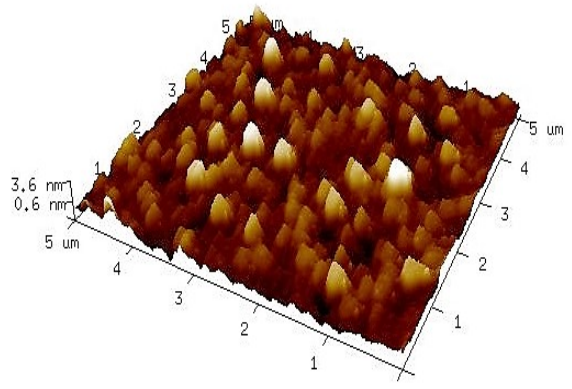

Figura 9. Imagen AFM 3D de la película delgada de nitruro de molibdeno depositada sobre silicio (111) a $400{ }^{\circ} \mathrm{C}$.

También medimos la rugosidad promedio $(\mathrm{Ra})$ y la rugosidad raíz media cuadrática (rms) de todas las muestras, usando el perfilador tridimensional, cuyos resultados se resumen en la tabla 4. En la figura 10 se muestra la variación de la rugosidad versus la temperatura de síntesis de las muestras de $\mathrm{Mo}_{2} \mathrm{~N}$.

Tabla 4. Valores de rugosidad promedio Ra y rugosidad eficaz Rq como función de la temperatura de sustrato.

\begin{tabular}{ccr}
\hline Temperatura $/{ }^{\circ} \mathrm{C}$ & $\mathrm{Ra} / \mathrm{nm}$ & $\mathrm{Rq} / \mathrm{nm}$ \\
\hline 100 & 1,093 & 1,343 \\
200 & 0,909 & 1,423 \\
300 & 0,457 & 0,618 \\
400 & 0,776 & 0,987 \\
\hline
\end{tabular}

La rugosidad promedio de la superficie depositada a $100{ }^{\circ} \mathrm{C}$ resultó ser de $1,093 \mathrm{~nm}$. Pero es reducida a $0,457 \mathrm{~nm}$ cuando la temperatura de deposición se incrementa a $300{ }^{\circ} \mathrm{C}$. Esta reducción en la rugosidad de la superficie puede estar relacionado con la reacción de los átomos de la superficie del molibdeno con los átomos de nitrógeno ${ }^{2}$. 


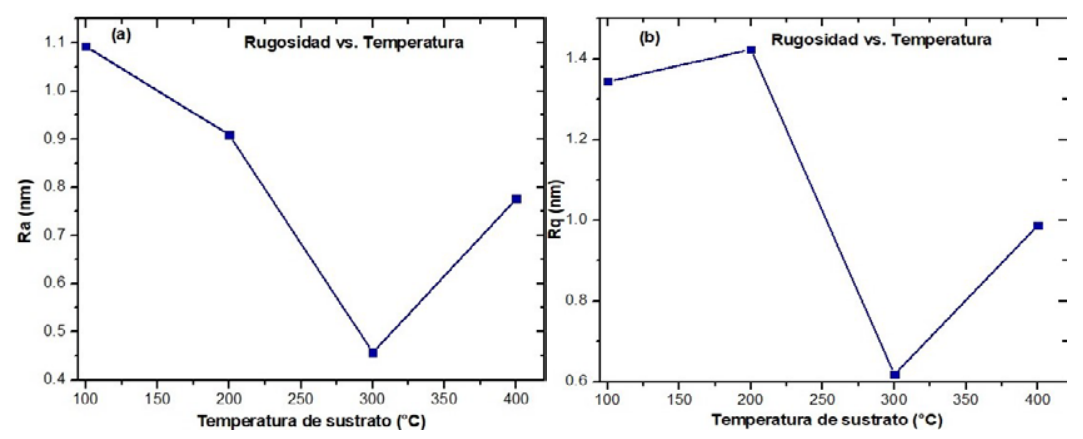

Figura 10. Rugosidad en función de la temperatura de sustrato. (a) Promedio, (b) rms.

La suavidad de la superficie es también particularmente atribuida al incremento de la velocidad de deposición, junto con la difusión y redeposición de átomos/iones de Mo después de la formación del nitruro; además, junto a la nitruración, puede mejorar la temperatura de la superficie localmente, lo que a su vez mejora la movilidad de los átomos de Mo en la superficie de la película en crecimiento, lo que también resulta en una película más suave ${ }^{20}$.

Luego, cuando se continúa aumentando la temperatura de síntesis a $400{ }^{\circ} \mathrm{C}$, la superficie rugosa se incrementa ligeramente a $776 \mathrm{~nm}$. Este aumento en la rugosidad puede atribuirse al incremento de la difusión superficial (movilidad) lo cual produce valles más grandes y más profundos ${ }^{2}$.

\section{CONCLUSIONES}

Se sintetizó satisfactoriamente las películas delgadas de nitruro de molibdeno sobre silicio (111) mediante pulverización catódica magnética reactiva dc en el rango de 100 a $400{ }^{\circ} \mathrm{C}$. El análisis de difracción de rayos $\mathrm{X}$ de las películas delgadas de $\mathrm{Mo}_{2} \mathrm{~N}$, indican que la temperatura de sustrato en el rango de 100 a $400{ }^{\circ} \mathrm{C}$ no influye significativamente en la estructura de tales películas. El tamaño de grano de las muestras se incrementa ligeramente con el aumento de la temperatura de sustrato, probablemente a la escasa difusión atómica a través del límite de grano. La resistividad de las películas de Mo2N disminuye con el incremento de la temperatura de síntesis, mostrando características óhmicas. Debido a la baja resistividad eléctrica y disminución de la rugosidad; estas películas pueden ser adecuadamente usadas como contacto óhmico posterior en células solares. 


\section{AGRADECIMIENTOS}

Este trabajo fue apoyado por la empresa PLANSEE SE, Reutte, Austria. Al Dr. Wilder Aguilar por la obtención de las imágenes AFM en el laboratorio de física de la Universidad Nacional de Trujillo. Los autores también agradecen a: José L. Ampuero por su apoyo en el análisis de las muestras por la técnica de espectroscopia electrónica Auger y a Junior D. Asencios por su apoyo en el laboratorio de sputtering de la Universidad Nacional de Ingeniería. A Fernando Hurtado por su apoyo en las mediciones eléctricas en el laboratorio de nanotecnología de la Universidad Nacional de Trujillo.

\section{REFERENCIAS BIBLIOGRÁFICAS}

1. Jauberteau I, Bessaudou A, Mayet R, Cornette J, Jauberteau JL, Carles P. Molybdenum Nitride Films: Crystal Structures, Synthesis, Mechanical, Electrical and Some Other Properties. Coat. 2015; 5(4): 656-687.

2. Khojier K, Mehr MRK, Savaloni H. Annealing temperature effect on the mechanical and tribological properties of molybdenum nitride thin films. J Nanostruct Chem. 2013; 3(1): 5. doi: 10.1186/2193-8865-3-5.

3. Sze P, Huang J, Chou D, Wang Y. Study of diffusion barriers for Au metal on liquid phase oxidized GaAs. J Vac Sci Technol B. 2006; 24(6): 2640-2644.

4. Thakur MK, Singh OP, Thakur K, Parmar R, Singh Gour K, Muhunthan N, et al. Effect of substrate on the structural and electrical properties of Mo thin films. Adv Mater Lett. 2016; 7(7): 525-528.

5. Guntur V. Molybdenum Nitride Films in the Back Contact Structure of Flexible Substrate CdTe Solar Cells. [Tesis para maestría]. Tampa, Florida: University of South Florida; 2011.

6. Senchenko E, Atuchin V, Khasanov T, Kochubey V, Pokrovsky L. Measurements of Optical Parameters of $\gamma$-Mo2N/Si (100) Thin Films. En: MEASUREMENT 2011, Proceedings of the 8th International Conference, Smolenice, Slovakia. 2011. p: 257-260.

7. Chen SF, Wang SJ, Lee WD, Chen MH, Wei ChN, Bor HY. Preparation and Characterization of Molybdenum Thin Films by Direct-Current Magnetron Sputtering. Atlas J Mater Sci. 2015; 2 (1): 54-59.

8. Miccoli I, Edler F, Pfnür H, Tegenkamp C. The 100th anniversary of the four-point probe technique: the role of probe geometries in isotropic and anisotropic systems. J Phys Condens Matter. 2015; 27(22):223201. doi: 10.1088/0953-8984/27/22/223201.

9. Hassan M, Qayyum A, Ahmad S, Naseer S, Khattak N, Zakaullah M. Structural and Mechanical Properties of Radiofrequency Ar-N2 Plasma Nitrided Aluminium. Mater Res. 2015; 18(2): 353-359.

10. Li M, Yang M, Vargas E, Neft K, Vanli A, Liang R. Analysis of variance on thickness and electrical conductivity measurements of carbon nanotube thin films. Meas Sci Technol. 2016; 27: 095004. doi: 10.1088/0957-0233/27/9/095004.

11. Vickerman J, Gilmore I. Surface Analysis. $2^{\text {nd }}$ edition. Chichester, UK: John Wiley \& Sons; 2009. 
12. Günzler H, Williams A. Handbook of Analytical Techniques. $1^{\text {st }}$ edition. Weinheim, Germany: Wiley-vch; 2001.

13. Kashyout AE-HB, Soliman HMA, Gabal HA, Ibrahim PA, Fathy M. Preparation and characterization of DC sputtered molybdenum thin films. Alexandria Eng J. 2011;50(1):57-63.

14. Shen L, Wang N. Effect of Nitrogen Pressure on the Structure of Cr-N, Ta-N, Mo-N, and W-N Nanocrystals Synthesized by Arc Discharge. J Nanomater. 2011; 2011:781935. doi: 10.1155/2011/781935.

15. Shen L, Cui Q, Zhang J, Li X, Zhou Q, Zou G. A New Method for Preparation of Nanocrystalline Molybdenum Nitride. Chin Phys Lett. 2005; 22: 3192. doi: 10.1088/0256$307 \mathrm{X} / 22 / 12 / 059$.

16. Rodríguez C. Variación de la resistencia eléctrica como función de la temperatura de sistema películadelgada de $\mathrm{SnO}_{2}$ - superconductor cerámico. [Tesis pregrado]. Trujillo: Universidad Nacional de Trujillo; 2015.

17. Srinivasan NB. MOCVD of Metal nitride thin films: Deposition, film characteristics and functionalproperties. [Tesis doctoral]. Bochum: Ruhr Universitat Bochum; 2014.

18. Anitha VP, Major S, Chandrashekharam D, Bhatnagar M. Deposition of molybdenum nitride thin films by r.f. reactive magnetron sputtering. Surf Coat Technol. 1996: 79: 5054.

19. Ahn H, Lee D, Um Y. Substrate Temperature Effects on DC Sputtered Mo thin film. 2017; Appl Sci Converg Technol. 26(1): 11-15.

20. Ikhlaq U, Ahmad R, Shafiq M, Saleem S, Shah MS, Hussain T, et al. Nitriding molybdenum: Effects of duration and fill gas pressure when using $100-\mathrm{Hz}$ pulse DC discharge technique. Chin Phys B. 2014; 23(10): 105203. doi: 10.1088/16741056/23/10/105203. 